\title{
Análisis del discurso de la competencia 27 del Currículo Nacional de la educación básica del Perú
}

\section{Analysis of the discourse of competence 27 of the National Curriculum of basic education of Peru}

\author{
Liz Alderete Callupe*I
}

*Departamento Académico de Educación, Facultad de Educación, Universidad Nacional del Centro del Perú (UNCP), Huancayo-Perú.

\begin{abstract}
Resumen
En este artículo se utiliza el método hermenoeducativo que corresponde a la investigación cualitativa, el análisis del discurso, específicamente el análisis de contenido. Para el análisis de contenido se utiliza el análisis semiótico constituido por el análisis sintáctico, semántico y pragmático, propio de las técnicas de análisis de las expresiones verbales escritas. Para interpretar el significado realde la competencia 27 del Currículo Nacional de la educación básica, que se imparte a través el área de educación para el trabajo en el nivel secundario de nuestro país. El currículo nacional de la educación básica (CNEB) es el documento normativo que rige la educación básica en el Perú. En este documento se encuentran las disposiciones a seguir en el sector de educación básica en los niveles: inicial, primaria y secundaria. En el CNEB se incluye el área de educación para el trabajo (EPT), en el que encontramos la competencia que busca desarrollar el área, esta competencia es el número 27, objeto de análisis de este estudio.
\end{abstract}

Palabras Claves: Competencia, educación para el trabajo, análisis de contenido, CNEB, educación.

\begin{abstract}
This article uses the hermenoeducational method of qualitative research discourse analysis, specifically content analysisto interpret the realmeaning of the competency 27 taughtthrough the area of education for work at the secondary level of regular basic education in our country. For content analysis, semiotic analysis is used, consisting of syntactic, semantic and pragmatic analysis, which is typical of the techniques of analysis of written verbal expressions. The national basic education curriculum is the normative document that containsthe provisions to be followed in the basic education sector. It includes the area of EFA, in which we find the competence that seeks to develop the area. This competence corresponds to number 27 , the object of analysisin this study.
\end{abstract}

Keywords: Competition twenty-seven, education for work, content analysis, CNEB.

1 Correspondiencia: Liz Alderete Callupe, liz.alderete.c@gmail.com 


\section{Análise do discurso da competência 27 do Currículo Nacional da educação básica do Perú}

\section{Resumo}

Este artigo se utiliza o método reivindicativo que corresponde à investigação qualitativa, a análise dodiscurso, especificamente a análise de conteúdo. Para a análise do conteúdo se utiliza a análise semiótico constituído pela análise sintático, semântico e pragmático, próprio das técnicas de análise das expressões verbais escritas. Para interpretar o significado real da competência 27 do currículo nacional da educação básica, que se transmite através da área de educação para o trabalho no nível secundário do nosso país. O currículo nacional da educação básica (CNEB) é o documento normativo que rege a educação básica no Peru. Neste documento se encontram as disposições a seguir no setor da educaçãobásica nos níveis: inicial, primaria e secundária. No CNEB se inclui a área de educação para o trabalho (EPT), é nele que encontramos a competência que busca desenvolver a área, esta competência é o número 27, objeto de análise deste estudo.

Palavras Claves: Competência, educação para o trabalho, análise de conteúdo, CNEB, educação.

\section{Kuskina chay competencia 27 del Curriculum Rimasqamanta mama llacctamanta chay edukasyun básica Perúnchikpa}

\section{Uchuycha}

Kayarticulupimimaynaruwasqan chay método hermenoeducativopaypam hucharaytataripay cualitativa nisqan, análisis rimasqanmanta, análisis nisqanmanta. Chaypinchanichin análisis semiótico constitutivo nisqanruwasqa chay análisis, sintáctco, semántico, pragmático nisqan, kikinpa chay técnica análisis chay simirimakunaqellqasqanisqan. Allinwillanakananpaq chay competencia iskaychunka qanchispi chay currículo de la edukasyunbásica, chaytamhun área edukasyunllamkana nivel secundario mama llaqtanchikpi. Chay curriculum nacional edukasyun básica chay documentumnormativumallinchanedukasyun básica Perú suyupi. Kaydicumentupitarikun disposiciones qatinapaqsectorpi chay edukasyun básica nivelpi: inicial, primaria y secundaria. Chay CNEB chaqtan chay área edukasyunllamkanapaq, chaypintarisuncompetenciata maskankururayta chay areapi, chay competenciam kan número iskaychunkaqanchispi numerupi, chaymikuskinayachaypa.

Hatun apup simi: Competenciam, yachay llamkanapa, kuskina conteniduta, CNEB, edukasyun. 


\section{Introducción}

La competencia número veintisiete se encuentra incluida en el Currículo Nacional de educación básica (CNEB). El CNEB es un documento emitido por el ministerio de educación del Perú, que se autodefine como orientador de los aprendizajes y establece qué aprendizajes deben lograr los estudiantes al culminar la educación básica. (Ministerio de educación del Perú, 2017)

Forma parte del CNEB la competencia número veintisiete que es el texto que se va a analizar. Se utilizará el método de análisis del discurso, específicamente el análisis de contenido porque forma parte de un documento escrito. El objetivo es descubrir el significado que encierra el texto que explica la competencia veintisiete, significado que no se da a conocer de manera explícita en el documento

Análisis del discurso educativo es un método de investigación social que corresponde al grupo metodológico hermenoeducativo. Sus procedimientos son: establecer unidades de análisis en el contexto, categorización de cada unidad, esquematización emergente, descripción sintáctica, semántica y pragmática, producción meta textual y esquematización. El objetivo que desea alcanzar es descubrir la importancia que el texto hablado o escrito tiene en la comprensión de la vida en la comprensión de la gestión de proyectos de emprendimiento económico o social (Martínez, 2004) (Cerrón, 2019). El análisis del contenido es una técnica que forma parte de la familia de las técnicas de análisis textual. Analiza el texto en sus tres dimensiones: sintáctica, semántica y pragmática. Busca encontrar lo que no está en el texto sino lo que está fuera de él. (Martínez, 2004)

Se denomina comúnmente corpus textual al texto que va a ser analizado (Martinez, 2004), para este estudio el texto o corpus textual forma parte de un todo que viene a ser el CNEB, el texto está ubicado en la página número 148 , siendo el siguiente:

\section{Competencia 27 Gestiona proyectos de emprendimiento económico o social}

Esta competencia se realiza cuando el estudiante lleva a la acción una idea creativa movilizando con eficiencia y eficacia los recursos, tareas y técnicas necesarias para alcanzar objetivos y metas individuales o colectivas con la finalidad de resolver una necesidad no satisfecha o un problema económico, social o ambiental. (Ministerio de Educación del Perú, 2017).

\section{Fase de análisis: Establecer las unidades de análisis en el contexto}

El primer paso para realizar la etapa de análisis es establecer las unidades de análisis en el contexto, las unidades de análisis o contenido, son segmentos de los datos narrativos para ir generando o descubriendo categorías que describan los conceptos de interés y sus vínculos, los cuales conforman el planteamiento del problema y permiten entender el fenómeno bajo análisis. (Hernández \& Mendoza, 2019).

Nuestra unidad de análisis es la competencia 27 del CNEB. Se eligió este texto porque el ministerio de educación resume en dicho escrito qué es lo que se quiere que los estudiantes logren en el área de educación para el trabajo:

Competencia 27 del currículo nacional de educación básica: Gestiona proyectos de emprendimiento económico o social.

Es cuando el estudiante lleva a la acción una idea creativa movilizando con eficiencia y eficacia los recursos, tareas y técnicas necesarias para alcanzar objetivos y metas individuales o colectivas con la finalidad de resolver una necesidad no satisfecha o un problema económico, social o ambiental.

La segunda etapa o fase de análisis es la categorización de cada unidad de análisis. Esto quiere decir que se debe poner un nombre breve que sintetice o resuma el significado de la unidad. (Martínez, 2004).

Tomando en cuenta la fase mencionada la unidad de análisis quedaría codificada como: Competencia 27 del CNEB. 
Las categorías establecidas son: Estudiante, acción, idea creativa, eficiencia y eficacia, recursos, tareas y técnicas, objetivos y metas, necesidad no satisfecha, problema económico, social o ambiental. A través de esta categorización se realizará el análisis a nivel semántico. Para hallar el significado real que se expresa en la competencia 27 del CNEB.

\section{Esquematización emergente}

El esquema es una técnica de representación de ideas. Utiliza gráficos, se escriben ideas breves y concisas, se organiza y clasifica jerárquicamente la información, así como se establecen relaciones lógicas entre las ideas. Permite tener una idea general del tema con tan solo leer el esquema.

Las categorías establecidas las representamos en un esquema. Se organizó la información de la misma manera como se presenta escrita la explicación de la competencia del área de educación para el trabajo en el CNEB.

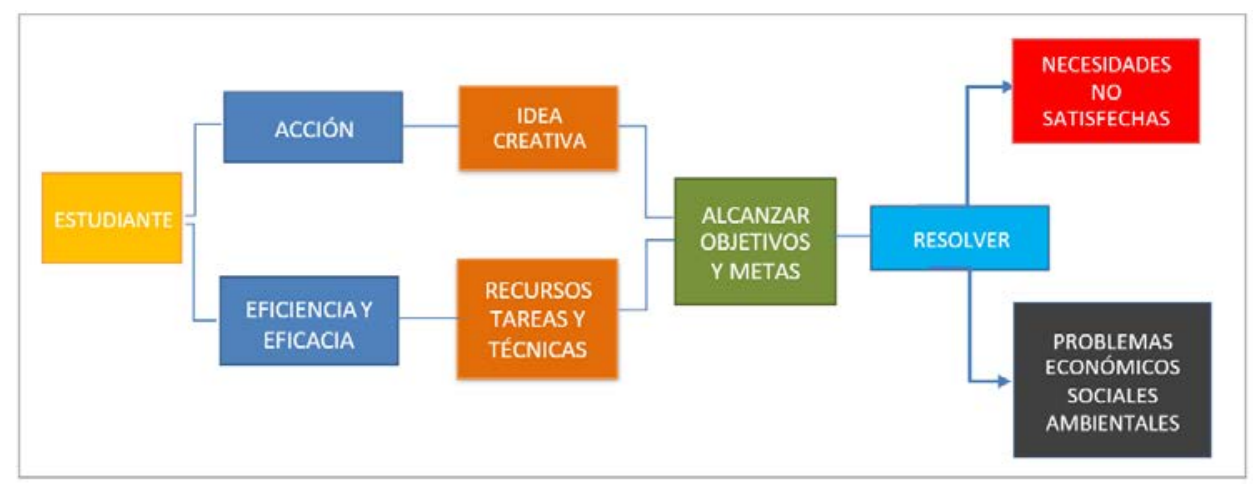

Figura 1. Esquematización de las categorías de la competencia 27 tal como se presentan en el CNEB.

\section{Descripción sintáctica}

Para Martínez Miguelez (2004), el análisis sintáctico está constituido por el análisis de la forma o superficie del texto. Se analizan las relaciones de concordancia y jerarquía entre los términos o palabras en su conjunto. El propósito es determinar si el texto está bien escrito, de ese modo la comprensión del texto será posible para el lector.

El texto al que vamos a realizar el análisis sintáctico, para este fin se vuelve a transcribir:

Competencia 27: Gestiona proyectos de emprendimiento económico o social.

Es cuando el estudiante lleva a la acción una idea creativa movilizando con eficiencia y eficacia los recursos, tareas y técnicas necesarias para alcanzar objetivos y metas individuales o colectivas con la finalidad de resolver una necesidad no satisfecha o un problema económico, social o ambiental.

El párrafo está constituido por una sola oración de 46 palabras, no está dividida en oración principal, secundaria y complementaria. La oración tiene dos verbos: "lleva a la acción" y "resolver". A través del verbo "lleva a la acción" entendemos que el sujeto de la oración es pasivo. El núcleo de la oración o sintagma nominal es: estudiante. En cuanto a número y persona está escrito en singular y género masculino. El predicado o sintagma verbal constituye la mayor parte de la oración con 38 palabras. Según el tipo de verbo, "lleva a la acción", la oración es del tipo predicativa. Se dice predicativa cuando el verbo denota una acción, en nuestro caso indica varias acciones. No presenta figuras como hipérbaton, alegorías, metáforas, debido a que forma parte de un documento oficial: el CNEB. (Vergara\& Liessem, 2013). El lenguaje utilizado para la redacción es formal, en tercera persona, que transmite un alto grado de formalidad.

El análisis sintáctico del texto de la competencia 27 por sí sola no bastaría para realizar el análisis de contenido, sin embargo, precede y se complementa con los otros dos análisis, el semántico y el pragmático. (Martínez, 2004) 


\section{Descripción semántica}

Descripción semántica, se podría decir que es la parte central del análisis del discurso porque tiene como objetivo hallar el significado o sentido del texto. (Martínez, 2004)

\section{Competencia 27: Gestiona proyectos de emprendimiento económico o social}

Es cuando el estudiante lleva a la acción una idea creativa movilizando con eficiencia y eficacia los recursos, tareas y técnicas necesarias para alcanzar objetivos y metas individuales o colectivas con la finalidad de resolver una necesidad no satisfecha o un problema económico, social o ambiental. Estudiante

Es la persona que estudia en el nivel de educación secundaria de la educación básica regular. Que se va formando a través del desarrollo de destrezas, habilidades, capacidades, competencias y valores. Para aprender moviliza conocimientos, los relaciona, realiza reflexión, vivencia experiencias diversas.

Por ello, lograr un proyecto elaborar e incluso gestionar un proyecto de emprendimiento pareciera una idea desmesurada que el estudiante no podría lograr, pero también debemos tener en cuenta que el estudiante en el nivel de educación básica regular se encuentra en proceso de formación, por ello, el significado por el cual nos inclinamos es que el estudiante es una persona que se está formando y aprenderá la competencia en el transcurso de su formación en el nivel secundario.

\section{Acción}

Es realizar algo. En nuestro contexto del análisis de la competencia 27, esa persona es el estudiante, que actúa por su voluntad de aprender. Para que el estudiante lleve adelante la competencia debe llevar a la acción diversos tipos de capacidades, habilidades que deberá combinar, acción desafiante que requerirá un asesoramiento claro de parte de su profesor o profesora.

\section{Idea creativa}

Es una idea diferente, novedosa y útil. Debe establecer una diferencia con otras ideas que ya existen. Sin embargo, en la realidad de la educación impartida, la creatividad una capacidad complicada de conseguir, debido al poco uso de estrategias de creatividad que tengan éxito. Por lo que más probable es que el estudiante genere ideas solo ideas novedosas.

\section{Eficiencia y eficacia}

Eficiencia es la capacidad de una persona para disponer de los recursos que tiene a su disposición de modo que consigue un efecto determinado. Eficacia es la capacidad de lograr el efecto que se desea o se espera, pero no necesariamente utilizando sustentablemente los recursos. Es evidente la diferencia entre ambas palabras. Escrito de esa manera en el texto en análisis, indica al estudiante que puede actuar de ambas maneras, cuando, debería estar solo la palabra eficacia y ya no considerar eficaz, porque al incluirlo estaría dando posibilidad de elegir la manera más rápida de actuar y no siempre el uso razonable de los recursos.

\section{Recursos}

Es un medio de distinto tipo, material, económico, humano que se tiene a disposición para desarrollar algo. Si la tarea principal del estudiante es realizar un proyecto de emprendimiento tendrá que utilizar diferentes recursos de acuerdo al tipo de proyecto de emprendimiento que va a crear. Estos recursos van a ser diferentes, de acuerdo a la especialidad en la que vaya a trabajar. En el caso de especialidades de producción de alimentos, vestimenta, muebles y otros similares, el costo de los recursos materiales es alto, y generalmente son los padres de 
familia de los estudiantes los que deben adquirirlos, ya sea por cada proyecto o con una contribución (cuota) económica al inicio del año. Muchas veces, la falta de recursos materiales y económicos limita el desarrollo de los proyectos. El ministerio de educación no dota de recursos para el área de educación para el trabajo, son las gestiones de las instituciones educativas e incluso de los docentes del área las que hacen posible la dotación de recursos, por esas razones nos preguntamos cómo se pide hacer uso eficiente de los recursos si no proveemos de los mismos.

\section{Tareas y técnicas}

La tarea es una actividad que se debe realizar, un trabajo. La técnica es un conjunto de procedimientos que ayudan a realizar alguna actividad específica. En el área de educación para el trabajo, el estudiante tendrá que "movilizar con eficiencia" tareas y técnicas. Será labor del docente enseñar a los estudiantes y de estos aprenderlas tareas o procedimientos que correspondan a los proyectos de emprendimiento y la aplicación de las técnicas de acuerdo a la especialidad con la que se esté trabajando: mecánica de metales, electricidad, computación, etc.

\section{Objetivos y metas}

Lo definimos como lo que se quiere alcanzar, la finalidad con que se dirigen las acciones o deseos de alguien. Acompaña a objetivos y metas las palabras individuales o colectivas. Interpretando el significado se puede manifestar que se sugiere que el proyecto, en primera instancia sea individual y como segunda opción sea colectivo. Privilegiando el trabajo en singular.

\section{Necesidad no satisfecha}

Es cuando una persona o varias no encuentran satisfacción o solución a una necesidad que le puede estar ocasionando problemas. El significado que se puede deducir es que se deben identificar las necesidades no satisfechas de los clientes, para crear o adecuar un producto que sí satisfaga aquellas necesidades o resuelva los problemas y el cliente consuma de inmediato el producto.

\section{Problema económico, social o ambiental}

Son problemas referidos a la falta de economía, escasez de recursos y a las necesidades generadas a partir de ellas. Los problemas sociales son condiciones que afectan a un gran número de personas, causando considerables inconvenientes y que según se cree debe corregirse mediante la acción social colectiva. El problema ambiental, es la perturbación, daño al entorno de la naturaleza. El ser humano es el responsable principal de este problema. (Real Academia Española, 2020)

Al mencionar en primer lugar a los problemas económicos interpretamos que está jerárquicamente adelante, se prioriza lo económico antes de los problemas sociales o ambientales.

Los significados considerados para cada categoría nos dan un panorama amplio del significado que no se muestra abiertamente en la competencia 27.

\section{Descripción pragmática}

A través de la descripción pragmática se debe identificar los usos, las prácticas y los propósitos, el para qué es usado el lenguaje escrito. (Martínez, 2004)

\section{Competencia 27: Gestiona proyectos de emprendimiento económico o social}

Es cuando el estudiante lleva a la acción una idea creativa movilizando con eficiencia y eficacia los recursos, tareas y técnicas necesarias para alcanzar objetivos y metas individual o colectivas con la finalidad de resolver una 
necesidad no satisfecha o un problema económico, social o ambiental.

Al leer el texto y buscar el sentido pragmático, encontramos que la primera parte nos indica que el estudiante lleva a la acción una idea creativa. Para ello deberá saber cómo generar una idea creativa, y no es solo eso, sino que debe realizar un uso eficiente y eficaz de los recursos, tareas y técnicas, es decir tres acciones, en la práctica el estudiante debe saber concebir una idea creativa, ser eficiente y debe ser eficaz. De esa manera conseguiría prioritariamente satisfacer una necesidad no satisfecha de alguien, contextualizado sería del cliente, y luego va, la posibilidad de resolver un problema económico, social o ambiental.

Aquí descubrimos que lo que se requiere es que el estudiante realice es identificar una necesidad que aún no se ha satisfecho, y, a partir de ella, desarrolle una solución por medio de la realización de un proyecto de emprendimiento, que vendría a ser un producto o servicio. Se menciona, además, que se debe tener en cuenta los problemas sociales y ambientales, pero siendo alternativas y no prioridades.

En resumen, de acuerdo al análisis pragmático lo que se expresa en el texto analizado es que el estudiante debe desarrollar un proyecto de emprendimiento económico. Sobre todo, identificando oportunidades en las necesidades del posible cliente. Para satisfacerlas a través del producto o servicio que ofrecerá.

En el área de educación para el trabajo, que en se inició con la denominación de educación técnica, se origina en los inicios del siglo veinte debido a la necesidad de las industrias de contar con trabajadores y artesanos técnicos, es decir mano de obra especializada; para el siglo 21 se ha variado el enfoque del área a formar personas que puedan emprender a través de proyectos y que estos proyectos, sobre todo económicos estén relacionados a las necesidades del cliente, de modo que en algún momento el estudiante debe ser capaz de emplearse o formar su propia empresa y gestionarla con éxito

Para determinar si en nuestro contexto cercano hay un resultado pragmático se realizó una encuesta. La encuesta constó de dos preguntas y estuvo dirigida a tres profesores del área de educación para el trabajo que tienen experiencia de más de ocho años en el desempeño como docentes del área en el ámbito de la provincia de Junín. Las preguntas fueron:

¿En la práctica conoce usted a algún estudiante que ha hecho su empresa? Podría describir brevemente.

¿Usted ha generado un proyecto de emprendimiento? ¿Por qué?

El primer profesor indicó que sí conoce estudiantes del distrito de Junín que han formado su empresa, como es el caso de los estudiantes S.V.H. y E.M.R quienes forman su empresa E\&S electricistas, brindando servicio de instalaciones eléctricas, instalaciones de redes, entre otras actividades.

A la segunda pregunta, el primer profesor dice que por la naturaleza del área los docentes estamos comprometidos con la generación de proyectos de emprendimiento y como una muestra a los estudiantes que sí se puede emprender aún en las dificultades. Donde prima la iniciativa, lo visionario que puede ser y con mucho optimismo mirando al futuro, empleando técnicas y herramientas de mercado.

A las mismas preguntas la segunda docente encuestada responde:

Si, un exalumno, junto con su familia, ha abierto una pequeña imprenta en la ciudad de Huancayo. Él empezó a trabajar en una empresa de diseño en cuanto terminó el colegio y ahí aprendió más sobre el diseño e imprenta y por eso puedo poner una tienda en ese rubro, lo que aprendió en el colegio en EPT le sirvió para que pueda iniciarse en el mundo laboral.

A la pregunta 2, menciona: “Con el anterior DCN con los alumnos de 5to grado hicimos la simulación para la constitución de una empresa desde la idea de negocio hasta la formalización de una empresa, cada alumno era dueño de una empresa formalizada. Todo quedó en simulación porque no se contaba con recursos para llevarlo a la práctica".

El tercer docente encuestado, en sus respuestas a las preguntas indica: "Respecto a la pregunta uno, estudiante que ha hecho su empresa, no, no conozco a ninguno, pero si muchos estudiantes que han salido a aplicar 
los aprendizajes en los negocios de sus padres, y muchos estudiantes que aprendían de negocio en el colegio, de vender productos como el yogurt de chirimoya y otros sabores que no hay en el mercado.

Acerca de la segunda pregunta, sí, había generado primero un taller de estampados, porque vi la necesidad de complementar con la microempresa de mi hermano y la gran necesidad que tenía él y muchos clientes. Tengo una papelería en Tarma, copias, etc. He generado porque en la Universidad del Centro y el Instituto pedagógico, que están cerca a mi casa (Pomachaca, Tarma), hacían monopolio, vendían a precio que los estudiantes no podían pagar. También inicié con impresión de sublimados plotter Epson, porque en campaña escolar los servicios de sublimados no atienden y no hay donde sublimar, fue una necesidad.

De las respuestas de los tres docentes encuestados del área de educación para el trabajo, se puede deducir que sí hay estudiantes que utilizan, en el sentido instrumental, práctico, los aprendizajes obtenidos a través del área de EPT en las instituciones educativas, para luego dar el siguiente paso que es iniciar un negocio y luego formar una empresa. En el caso de generación de proyectos de emprendimiento de los mismos docentes, solo el docente tres expresa que sí tiene una empresa en conjunto con su hermano. Inició las actividades de emprendimiento debido a la necesidad del mercado, lo que coincide con el texto analizado en el sentido que, en este caso la finalidad fue resolver una necesidad no satisfecha de los clientes aprovechando la oportunidad que se presentó en su entorno.

\section{Producción meta textual}

Luego de haber realizado los análisis de contenido en los tres niveles: sintáctico, semántico y pragmático se puede entender que a pesar que el texto que explica la competencia 27, incluye dentro de sí una intención de desarrollar proyectos que ayuden a resolver problemas sociales y ambientales, en realidad le da prioridad al desarrollo de proyectos económicos, porque tiene predominio dentro del texto que se encuentra en el CNEB, el documento que rige la educación básica escolar peruana, de ese modo, como en sus inicios, la enseñanza técnica, ahora el área de educación para el trabajo se rige bajo los preceptos de la economía de mercado que está asociada principalmente a la producción y consumo de bienes sin tener en cuenta el impacto que puede causar, ahora más evidente son las consecuencias que están presentes al estar experimentando una pandemia.

Por tanto, a mi parecer, como docente del área propongo que se cambie el enfoque y la competencia del área de EPT, dando prioridad al desarrollo social, ambiental que daría paso a un desarrollo económico más equitativo y sostenible con el uso racional de los recursos, no solo con la idea de obtener ganancia económica inmediata sino de forjar un futuro sostenible para la sociedad y la conservación del medio ambiente, lo que implicaría modificar las estructuras mentales tanto de las autoridades, comunidad educativa y sociedad en general.

\section{Esquematización}

De acuerdo propuesta realizada, las categorías del discurso educativo condensado en la competencia número 27 debe quedar como se muestra en la figura número 2, consideramos más sostenible y equitativo para el desarrollo de proyectos que a la larga van a beneficiar a la mayoría y no solo al que lo produce.

En el esquema se representa la propuesta que consideramos más pertinente.

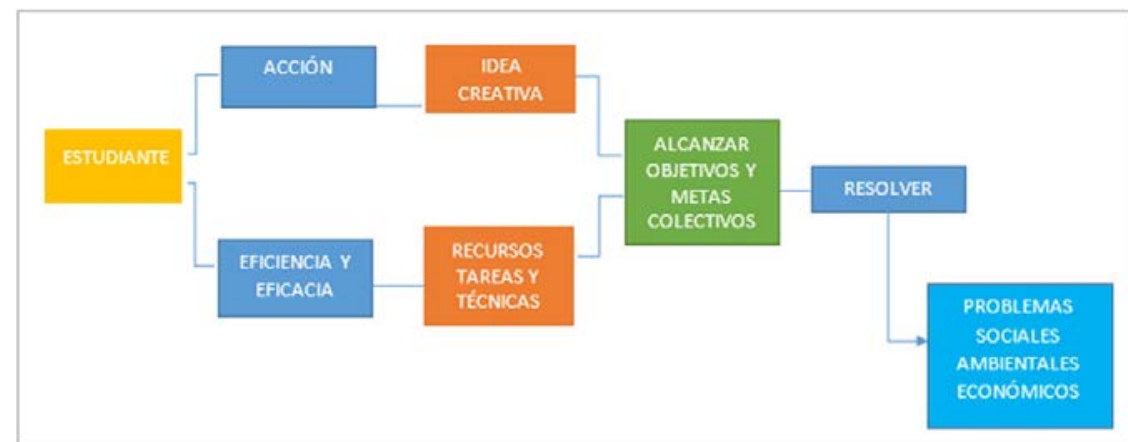

Figura 2. Propuesta de una nueva esquematización que prioriza un desarrollo sostenible. 


\section{Materiales y métodos}

El método utilizado para analizar fue el análisis del discurso, utilizado en las investigaciones cualitativas, como la competencia veintisiete forma parte del Currículo Nacional, es un documento escrito, por lo que se eligió el análisis del contenido, para analizar expresiones verbales, en este caso escritas en este documento cuyo discurso es de carácter educativo.

El análisis de contenido sigue los siguientes procedimientos: establecer las unidades de análisis, categorizar cada unidad de análisis, elaborar una esquematización emergente, realizar la descripción del texto a través de los niveles sintáctico, semántico y pragmático, realizarla producción meta textual y a partir de ella la nueva esquematización. (Martínez, 2004) (Cerrón, 2019)

Para el análisis pragmático se realizó preguntas tipo encuestas a tres profesores del área de educación para el trabajo, cuya característica principal es que tienen experiencia de más de 8 años en la enseñanza del área. Sus respuestas ayudaron a confirmar el sentido práctico que encierra el texto analizado.

\section{Discusión de resultados}

Al realizar el análisis del contenido de la competencia 27 del CNEB, se ha encontrado que el texto que explica la competencia en realidad encierra dentro de sí un significado diferente al que aparentemente da. En el texto que tenemos ahora, la competencia 27 del CNEB, el estudiante se preocupa por otra persona, representada por el cliente, al decir que se resolverá una necesidad no satisfecha, en realidad se refiere a aprovechar una necesidad presente en el mercado con el objetivo de obtener una ganancia económica, a la vez que el emprendedor, en este caso el estudiante, a lo largo de su proceso de formación en la educación secundaria aprenderá a priorizar solo a aprovechar oportunidades para vender. No es eso lo que se debe enseñar o lo que el estudiante debe aprender, sino, acorde al proceso reflexivo el estudiante debe aprender a desarrollar proyectos que beneficien a su localidad, a su entorno cuidando en ese proceso el medio ambiente y generando bienes y servicios destinados al bien común.

\section{Conclusiones}

La competencia veintisiete del CNEB tiene un significado diferente al ser analizado en los tres niveles de análisis semiótico, en el que el estado a través del ministerio de educación, hace prevalecer la economía de mercado en detrimento de la formación para el desarrollo de proyectos de emprendimiento que prioricen la solución de problemas sociales y ambientales, que posibilitarían una de las principales razones por las que en este momento estamos viviendo en situación de crisis y gran desigualdad.

Los tres niveles de análisis: sintáctico, semántico y pragmático se complementan para hallar el verdadero significado de la competencia veintisiete del CNEB, descubierto al interpretarlas unidades de análisis y el texto.

Al realizar el análisis pragmático con la interpretación de las respuestas brindadas por tres docentes del área de educación para el trabajo se confirma que la principal intención de la competencia es que el estudiante se especialice en el sentido práctico, técnico y de uso de recursos para desenvolverse en algún rubro o crear una empresa solo en base a esos factores. 


\section{Referencias Bibliográficas}

Cerrón, W. (2019). La investigación cualitativa en educación. Horizonte de la Ciencia, 159-167.

EDUCREA. (28 de 07 de 2020). Obtenido de https://educrea.cl/importancia-de-las-tic-en-laeducacion-basica-regular/

Hernández, R., \& Mendoza, C. (2019). Metodología de la investigación. Las rutas cuantitativa, cualitativa y mixta. Mc Graw Hill Education.

Martínez, M. (2004). Ciencia y arte en la metodología cualitativa. Trillas.

Ministerio de educación del Perú. (2017). Currículo nacional de la educación básica. Ministerio de educación

Navarro, P., \& Díaz, C. (1994). Métodos y técnicas cualitativas de investigación en Ciencias Sociales. En P. Navarro, \& C. Díaz. Síntesis

Real Academia Española. (31 de Julio de 2020). Diccionario de la lengua española. Obtenido de https://dle.rae.es/ contenido/cita

Vergara, E., \& Liessem, M. (2013). Descubriendo la sintaxis. Ministerio de educación, cultura y deporte de España. 